\title{
BDSG 2.0
}

Ein Vierteljahrhundert liegt die Veröffentlichung des „Volkszählungsurteils" des Bundesverfassungsgerichts vom 15.12.1983 zurück. Liest man die Urteilsbegründung heute, so mutet sie geradezu seherisch an:„Die Möglichkeiten der modernen Datenverarbeitung sind weithin nur noch für Fachleute durchschaubar und können beim Staatsbürger die Furcht vor einer unkontrollierbaren Persönlichkeitserfassung selbst dann auslösen, wenn der Gesetzgeber lediglich solche Angaben verlangt, die erforderlich und zumutbar sind.“ Und:„Individuelle Selbstbestimmung setzt aber - auch unter den Bedingungen moderner Informationsverarbeitungstechnologien - voraus, daß dem Einzelnen Entscheidungsfreiheit über vorzunehmende oder zu unterlassende Handlungen einschließlich der Möglichkeit gegeben ist, sich auch entsprechend dieser Entscheidung tatsächlich zu verhalten.“ Schließlich:„Hieraus folgt: Freie Entfaltung der Persönlichkeit setzt unter den modernen Bedingungen der Datenverarbeitung den Schutz des Einzelnen gegen unbegrenzte Erhebung, Speicherung, Verwendung und Weitergabe seiner persönlichen Daten voraus."

Diese Feststellungen sind nicht nur immer noch gültig und aktuell. Wichtiger noch: Man muss sie verstehen vor einem Kontext "moderner Informationsverarbeitungstechnologien", die heute steinzeitlich anmuten. 1983 gab es in Deutschland kein Rechenzentrum, dass die Leistungsfähigkeit und Speicherkapazität eines heute handelsüblichen PCs besaß. Die Intel 80286-CPU der PCs von 1983 bestand aus 134.000 mit $8 \mathrm{MHz}$ getakteten Transistoren. In einer Dual Core Itanium 2-CPU eines heutigen PCs werkeln 1,7 Milliarden Transistoren, also $12.600 \mathrm{mal}$ so viele, getaktet mit $1,6 \mathrm{GHz}$, also $200 \mathrm{mal}$ so schnell. Für dieselbe Rechenleistung hätte man 1983 2,5 Millionen PCs benötigt fast den gesamten Weltmarkt. Und auch die Speicherkapazität ist explodiert: „Terrabyte" waren vor 25 Jahren eine schier unvorstellbare Größenordnung. Die damals gerade erschienenen ersten PC-Festplatten fassten 10 MByte - 200.000 Exemplare wären zur Speicherung von zwei Terrabyte Daten erforderlich gewesen, der Speicherkapazität einer heutigen, gerade einmal acht Quadratzentimeter großen SDXD-Karte. Vor allem aber waren Rechenzentren und PCs 1983 nicht vernetzt, sieht man von (teuren) Wählverbindungen mit 300 bit/s ab, über die man gezielt einzelne Dateien übermitteln oder über ein Terminalprogramm auf Großrechner zugreifen konnte. Heutige private DSLAnschlüsse erlauben Datenübertragungen mit bis zu $52 \mathrm{MBit} / \mathrm{s}$ - das 175.000fache.

Diese Entwicklung und das Internet als eine heute nahezu jedermann zugängliche, gigantische internationale „Wissensdatenbank“ haben erhebliche Auswirkungen auf den Datenschutz. Die Folgen von Defiziten in der Umsetzung des BDSG, die es schon immer gab, haben nun eine andere Dimension. Auch gebiert das Internet neuartige Dienste wie Social Networks, die im Kern in der (mehr oder weniger ungezielten) Verbreitung personenbezogener Daten bestehen. Und mit der zunehmenden Bedeutung des Internet für zahlreiche Geschäftsprozesse wachsen auch die Begehrlichkeiten nach Nutzerdaten.

Angesichts dieser erheblich veränderten technischen und sozialen Rahmenbedingungen der automatisierten Datenverarbeitung verwundert die Zurückhaltung der Politik. Die Regulierung des Schutzes personenbezogener Daten, Ausfluss des Rechts auf freie Entfaltung der Persönlichkeit (immerhin Art. 2 GG) basiert noch immer auf den Rahmenbedingungen der späten 80er Jahre des vergangenen Jahrhunderts. Die überfällige Modernisierung des Datenschutzrechts steht in krassem Kontrast zu einer Innenpolitik, die im Windschatten der Bedrohung durch internationale Terroristen mit Vorratsdatenspeicherung und Onlinedurchsuchung den Rahmen des verfassungsrechtlich Zulässigen aus- und gelegentlich überreizt. „Wer nicht mit hinreichender Sicherheit überschauen kann, welche ihn betreffende Informationen in bestimmten Bereichen seiner sozialen Umwelt bekannt sind, und wer das Wissen möglicher Kommunikationspartner nicht einigermaßen abzuschätzen vermag, kann in seiner Freiheit wesentlich gehemmt werden, aus eigener Selbstbestimmung zu planen oder zu entscheiden", formulierten die Verfassungsrichter 1983. Dem ist auch im Jahr 2009 nichts hinzuzufügen.

\section{Dirk Fox}

